\title{
CARACTERIZAÇÃO DOS DESAFIOS PARA O DESENVOLVIMENTO LOCAL DE NOVOS PRODUTOS DO SETOR FARMACÊUTICO: UMA VISÃO DA RELAÇÃO UNIVERSIDADE - EMPRESA NO ECOSSISTEMA MINEIRO
}

Marina Bastos Carvalhais Barroso (marinabastosb@gmail.com) - Universidade Federal de Minas Gerais

Beatriz Amâncio Sofal (beatrizsofal@yahoo.com) - Universidade Federal de Minas Gerais

Letícia Carvalho Santana Rocha (letycsr@gmail.com) - Universidade Federal de Minas Gerais

Tiago Paz Lasmar (tplasmar@gmail.com) - Universidade Federal de Minas Gerais

Raoni Barros Bagno (rbagno@,dep.ufmg.br) - Universidade Federal de Minas Gerais

\section{RESUMO}

Dada importância do setor farmacêutico, é latente a necessidade de consolidação local de competências que suportem o desenvolvimento de novos produtos. Neste contexto, relações universidade-empresa são ponto chave para inovar no setor. Entretanto, complexidades inerentes à dinâmica de inovação típica da indústria farmacêutica e sua trajetória tecnológica local intensificam de forma especial este desafio. O desenvolvimento de inovações no setor é caracterizado por ser um processo longo, de capital intensivo, fortemente dependente de $P \& D$ e cercado de incertezas. Tal processo pode ainda perpassar vários agentes de uma complexa cadeia produtiva, com fortes assimetrias em termos de norteamento estratégico e domínio tecnológico. Focado nas relações entre universidade e empresa, este estudo tem o objetivo de identificar e discutir lacunas existentes no desenvolvimento de novos fármacos. Para isso, a análise conta com a aplicação de entrevistas semiestruturadas a atores chaves na relação universidade-empresa, incluindo professores universitários, um representante do NIT de uma universidade e profissionais de empresas do setor. $O$ trabalho oferece um diagnóstico inicial que relaciona as possíveis barreiras da relação universidadeempresa sob o ponto de vista de diferentes atores da cadeia do setor farmacêutico. Ademais, evidencia pontos críticos de possiveis ações que auxiliem no enfrentamento dos aspectos contingenciais deste ecossistema e no robustecimento de uma abordagem de gestão da inovação no setor farmacêutico.

Palavras-chave: Gestão da inovação; relação universidade-empresa; setor farmacêutico

Área: Gestão do Processo de Desenvolvimento de Produtos

\section{INTRODUÇÃO}

A inovação tecnológica, desde Schumpeter (1934), surge como rotina essencial para se alcançar e sustentar vantagens competitivas em novos mercados e diferentes setores. $\mathrm{O}$ desenvolvimento de inovações na indústria farmacêutica é caracterizado por ser um processo longo, de capital intensivo, fortemente dependente de P\&D e cercado de incertezas de variadas naturezas. Segundo Bastos (2005), os gastos em P\&D farmacêutico superam os recursos destinados por outras indústrias.

Albuquerque e Cassiolato (2002) enfatizam as diferenças intersetoriais nas relações entre ciência, tecnologia e produção, fatores chaves para o desenvolvimento de inovações. Sendo 
assim, a dinâmica da inovação está diretamente relacionada ao setor que se busca essa vantagem competitiva, à medida que uma série de fatores que impactam nessa dinâmica, como financeiros, políticos, tecnológicos e regulatórios, são setorialmente dependentes. Dada a sua expressão econômica e social e complexidades inerentes à dinâmica de inovação, o setor farmacêutico tem o desafio de desenvolvimento de produtos intensificado. Neste contexto, para dar suporte às empresas no desenvolvimento de tecnologia, emerge o conceito de colaboração interorganizacional, no qual a cooperação universidade-empresa é uma de suas formas mais usuais (CASTILHO et al., 2015).

Focado nas relações entre universidade e empresa, este estudo tem o objetivo de identificar e discutir lacunas existentes no desenvolvimento de novos fármacos. Especificamente, busca entender sob a perspectiva de diferentes atores da cadeia farmacêutica, quais são os principais desafios enfrentados para a construção da relação universidade-empresa de forma efetiva. Para isso, a análise conta com entrevistas semiestruturadas aplicadas a pesquisadores, representantes de empresas públicas e privadas do setor farmacêutico, além da representante do Núcleo de Inovação Tecnológica (NIT) de uma universidade pública, todos estes localizados no estado de Minas Gerais.

O artigo está organizado da seguinte forma: o referencial teórico aborda a relação universidade-empresa, seguido das particularidades do setor farmacêutico. Depois, as etapas da coleta de dados do trabalho são descritas na metodologia, e então apresentam-se os resultados alcançados. Por fim, são apresentadas as conclusões da pesquisa e suas limitações.

\section{REVISÃO TEÓRICA}

\subsection{Relação universidade-empresa}

A relação de cooperação entre a universidade e os setores produtivos destaca-se atualmente como uma das formas mais difundidas para a promoção do desenvolvimento tecnológico e de inovações. Nesse sentido, é necessário que se busque uma maior interação entre essas instituições estimuladas por mecanismos que ampliem os benefícios bilaterais desse vínculo.

Webster e Etzkowitz (1991) apontam razões que motivam a ampliação da interação universidade-empresa. Para as empresas, há a indispensabilidade de inovar constantemente para se manter em um mercado extremamente competitivo. Para a universidade, é característico o desafio de obtenção de recursos para o desenvolvimento de pesquisas e legitimação de seu papel social. Ainda, segundo Lopéz-Martinéz et al. (1994 apud SEGATTO-MENDES, 1996, p. 10), em países em desenvolvimento essa interação é uma maneira de modernizar os parques industriais, por estabelecer parcerias com universidades para em médio prazo obter capacidade de $\mathrm{P} \& \mathrm{D}$ própria.

Etzkowitz (2008) enfatiza a necessidade de conexões interorganizacionais ao afirmar ser a Tríplice Hélice, interação entre universidade-indústria-governo, a chave para inovação em sociedades cada vez mais baseadas em conhecimento. O principal papel do governo é o de fomentar essa interação, principalmente por meio de políticas públicas. Essas políticas têm aumentado incentivos para a cooperação entre universidade-indústria, por considerá-la crucial para o desenvolvimento tecnológico no país (HASENCLEVER, 2013).

Apesar da importância supracitada, há obstáculos para a efetividade da relação universidadeempresa. O estudo de Bruneel, d'Este e Salter (2010) apresenta estes desafios em dois tipos de barreira distintas: aquelas relacionadas às diferenças entre as orientações da indústria e universidade e aquelas relacionadas à conflitos sobre propriedade intelectual e à administração universitária - barreiras de transação. 
Em relação às barreiras relacionadas à orientação, é primordial considerar devidamente as diferenças na dinâmica de trabalho de cada instituição, interesses centrais das pesquisas, visão estratégica e visão de longo versus curto prazo (CASTILHO et al., 2015). Nas universidades os acadêmicos têm as publicações como um forte mecanismo de incentivo, o que pode resultar em pesquisas que não possuam necessariamente aplicação prática e interesse do mercado. Segatto-Mendes (1996) enfatiza do ponto de vista da orientação o foco da empresa em obtenção de lucro. Dessa forma, quanto maior a incerteza de retorno do investimento, menor o interesse da empresa no projeto.

As barreiras relacionadas à transação, por sua vez, são causadas pelas diferentes noções de confidencialidade. As empresas utilizam conhecimento para ganhar vantagem competitiva, e, portanto, o conhecimento "privado" é fechado, permanecendo sigiloso ou limitado por patentes (PARTHA; DAVID, 1994). Já os pesquisadores tendem a divulgar informações para obter reconhecimento e fomentar o desenvolvimento científico.

Por fim, há diferenças na comunicação entre pessoas da universidade e da empresa. Enquanto a linguagem é científica do lado da universidade, é voltada a negócios no lado empresarial. Esses aspectos impactam diretamente na operação dos NITs das universidades.

\subsection{Características da indústria farmacêutica}

A indústria farmacêutica é cercada de singularidades. Caracterizada como intensiva em pesquisa e ciência, e com grande importância social e econômica, requer um foco de destaque em atividades de P\&D (PAVITT, 1984). Tal processo pode perpassar por vários agentes da cadeia produtiva, com fortes assimetrias em termos de norteamento estratégico e domínio tecnológico. Conforme afirma Bastos (2005), o processo de inovação farmacêutica envolve o relacionamento de empresas com outras instituições, como a própria universidade, a fim de lançar produtos novos ou modificados.

O setor farmacêutico evoluiu em um ritmo acelerado desde o século XIX, passando por diversas fases de mudança tecnológica. Segundo Radaelli (2008), essa evolução atravessa três ondas tecnológicas desde a produção artesanal de medicamentos, passando pelo desenvolvimento da química orgânica e compostos microbiológicos, até o advento da biotecnologia e recentemente a nanotecnologia. Essa alteração no caráter tecnológico da produção de fármacos passou a exigir uma base científica consolidada, com gastos volumosos em pesquisas para desenvolvimento de inovações no setor. Contudo, esses elevados gastos não significam alto desempenho em inovação. Em média, apenas 1 dentre 10000 substâncias tornam-se um produto possível de mercado, e apenas um terço das novas drogas gera receita que supera o gasto médio em P\&D da empresa (GRABOWSKI et al., 2002).

No setor, o insucesso de projetos só é detectado em estágios clínicos avançados, após grandes esforços de desenvolvimento e altos gastos (GASSMANN et al., 2008). Tipicamente, a validação da pesquisa passa por duas grandes fases: os testes pré-clínicos, com duração de três a seis anos; e os ensaios clínicos distribuídos em três fases, com duração total de seis a nove anos (PALMEIRA FILHO; PAN, 2003). A partir dessa perspectiva, a propriedade intelectual torna-se altamente relevante para estímulo da inovação farmacêutica. Os lucros de monopólio assegurados pelos direitos de patente durante seu prazo de vigência reforçam as possibilidades de que a empresa recupere o investimento realizado.

Contudo, direitos de propriedade intelectual não perfazem a única questão regulatória. Órgãos reguladores têm grande papel na realidade do setor farmacêutico, incluindo questões de regulação de preço, regras dos sistemas públicos de saúde, diretivas aplicadas ao processo de P\&D e homologação de processos de produção. No Brasil, a Agência Nacional de Vigilância 
Sanitária (ANVISA, 2017) é a responsável pelas atividades de regulação, normatização, controle e fiscalização farmacêutica.

Dados do Sindusfarma (2016), fundamentados em pesquisas do IMS Health, mostram que nos últimos 15 anos a indústria de capital estrangeiro representou, em média, cerca de $60 \%$ do total de vendas no mercado brasileiro de medicamentos. No contexto brasileiro, em sua maioria, as empresas farmacêuticas são de menor porte e a participação de empresas de base biotecnológica, formadas a partir de spin-offs acadêmicos, é crescente (BASTOS, 2005). Contudo, a participação de empresas de capital nacional nesse segmento vem se tornando cada vez maior, principalmente após a Lei dos Medicamentos Genéricos (9.787/1999). Vale dizer que o desenvolvimento destes medicamentos requer um investimento significativamente menor do que um novo medicamento (BITTENCOURT et al., 2012). Conforme Pimentel et al. (2013), o investimento relativo em P\&D pelas empresas brasileiras é baixo, e as multinacionais mantêm suas atividades de $\mathrm{P} \& \mathrm{D}$ e produção de farmoquímicos no exterior. De modo geral, no Brasil, atividades dessa natureza se restringem às universidades e instituições públicas de pesquisa, não havendo tradição de parceria entre esses agentes e a indústria (DA MOTTA VIEIRA; OHAYON, 2006).

\subsection{Relação universidade-empresa no setor farmacêutico}

A relação universidade-empresa recebe destaque quando se considera o setor farmacêutico. Levando em consideração o importante papel desempenhado pelas universidades como fonte primordial de geração de conhecimento (BITTENCOURT et al., 2012), percebe-se a relevância desse tipo de relação para o setor. Contudo, as barreiras existentes a essa interação estão presentes no setor com as suas especificidades. "Enquanto na empresa o principal objetivo é a satisfação dos interesses dos proprietários, na universidade o interesse está na realização das necessidades sociais." (SEGATTO-MENDES, 1996). Exemplo desse conflito é a busca por soluções para doenças negligenciadas, aquelas que possuem forte incidência em países de baixa renda; e órfãs, que atingem parcelas ínfimas da população mundial. Pelo seu baixo potencial mercadológico, essas doenças não despertam o interesse típico da indústria, que se volta a doenças como o câncer e a hipertensão.

Os diferentes objetivos dessas organizações em conjunto com a intensa regulação setorial impactam na dinâmica de validação das tecnologias e, consequentemente, tornam-se uma barreira na relação efetiva dessas entidades. Isso porque os procedimentos de validação exigidos para elaboração de uma publicação acadêmica não são suficientes para a comprovação de eficiência de produção e legislativa para a indústria. Por exemplo, os testes pré-clínicos realizados nas ICTs não têm validade regulatória se não forem realizados sob certificação de boas práticas laboratoriais (PARANHOS, 2012). Ainda segundo a autora, no Brasil as dificuldades são ainda maiores pela ausência de infraestrutura para desenvolvimento experimental, o que é essencial para ligar a pesquisa científica (bancada) com a industrial.

A barreira de transação é presente com maior expressividade no setor farmacêutico. A principal reclamação das empresas é a falta de regras claras e de meios diretos de estabelecer de contratos com as ICTs (PARANHOS, 2012). Considerando a importância da propriedade intelectual para o setor, quando a obtenção de patente, por exemplo, torna-se um gargalo, o restante do desenvolvimento é travado. A figura 1 apresenta a síntese das barreiras da relação universidade-empresa mapeadas na literatura. 
Figura 1. Barreiras da relação universidade-empresa mapeadas na literatura. Fonte: Elaborada pelos autores (2017)

\begin{tabular}{|c|l|c|}
\hline \multicolumn{3}{|c|}{ Barreiras relacionadas à orientação } \\
\hline Barreira & Contexto do setor farmacêutico & Referência \\
\hline Divergência de objetivos & $\begin{array}{l}\text { Atuação das universidades com } \\
\text { doenças de interesse social e da } \\
\text { indústria com doenças de maior } \\
\text { apelo financeiro. }\end{array}$ & $\begin{array}{c}\text { (SEGATTO- } \\
\text { MENDE, 1996) }\end{array}$ \\
\hline Validação da tecnologia & $\begin{array}{l}\text { A validação de pesquisa para fins } \\
\text { de publicação acadêmica não } \\
\text { atende aos requisitos regulatórios } \\
\text { para produção industrial. }\end{array}$ & (PARANHOS, 2010) \\
\hline Barreiras relacionadas à transação \\
\hline confidencialidade & $\begin{array}{l}\text { Contexto do setor farmacêutico } \\
\text { publicar informações para obter } \\
\text { reconhecimento enquanto as } \\
\text { empresas as querem em sigilo para } \\
\text { vantagem competitiva. }\end{array}$ & Referência \\
\hline Comunicação e nível de \\
conhecimento
\end{tabular}

\section{METODOLOGIA}

A coleta de dados se deu por meio de entrevistas semiestruturadas com atores na relação universidade-empresa no setor farmacêutico. Buscou-se a visão do desafio da aproximação dessas instituições sob diferentes perspectivas. Para tanto, entrevistou-se pesquisadores na área farmacêutica, representantes de empresas do setor, além de uma representante do Núcleo de Inovação Tecnológica (NIT) de uma universidade pública. A partir dessa definição, foram realizadas duas entrevistas piloto, objetivando uma maior imersão no ambiente estudado e entendimento das práticas recorrentes no setor farmacêutico, especificamente o mineiro. Após estas primeiras entrevistas, foi possível a estruturação de um protocolo mais formal para ser usado em outras cinco entrevistas.

Inicialmente, procurou-se entender o histórico de envolvimento do agente com a temática, a partir da sua trajetória, funções organizacionais e atuação no setor. Na sequência a pergunta chave da pesquisa era feita: "quais são as principais oportunidades e barreiras da relação universidade-empresa no setor farmacêutico?". Por fim, ao final da entrevista, foram levantados pontos de ação futura por meio do questionamento de quais iniciativas seriam necessárias para superar os desafios do contexto dessa relação.

De modo a permitir uma melhor análise dos dados obtidos, as barreiras destacadas por cada entrevistado foram mapeadas e foi verificada sua aderência ou não com aquelas encontradas 
na literatura. Isso motivou a consideração de novas barreiras. A visão da universidade foi coletada por meio da entrevista com três pesquisadores, membros de uma rede mineira de pesquisa em nanobiotecnologia. Já a visão da indústria foi captada pela entrevista com dois agentes distintos. O primeiro atua em associação das indústrias farmacêuticas e é sócio de uma empresa do setor, possuindo ainda experiência na ANVISA. O segundo é uma gerente de uma indústria de fitoterápicos. A quarta instituição envolvida é uma fundação pública que tem três frentes de atuação: pesquisa, produção e vigilância. Por fim, foi entrevistada a coordenadora do NIT. Sendo este o núcleo responsável na universidade por intermediar a relação com as empresas, julgou-se relevante entender quais os principais desafios enfrentados nesse órgão.

\section{RESULTADOS}

Identificou-se em termos gerais que os desafios apontados pela literatura se manifestam na prática, mas identificaram-se também outros novos. Cada barreira se manifestou de forma diferente, de acordo com a perspectiva de cada entrevistado e algumas obtiveram destaque.

\subsection{Barreiras relacionadas à orientação}

Dentre todos os aspectos reportados relativos à relação universidade-empresa, a grande maioria se agrupa na barreira que diz respeito à divergência de objetivos dessas duas instituições. Essa barreira se manifestou em todas as entrevistas realizadas.

Levantou-se que os pesquisadores muitas vezes desenvolvem pesquisas fora da vocação da indústria e com pouca viabilidade de aplicação. Por esse motivo tais projetos não despertam interesse do mercado. De fato, segundo um dos pesquisadores, a definição da linha de pesquisa é, na maioria das vezes, escolha unilateral do grupo de pesquisadores, não levando em consideração uma aproximação e mapeamento de demanda com a indústria. A principal justificativa para a falta de alinhamento do portfólio de pesquisa estaria no papel social da universidade. Os representantes da fundação, afirmaram que há uma visão negativa dentro das instituições públicas quanto ao estabelecimento de parcerias universidade-empresa. Como membros do sistema público, os pesquisadores deveriam se preocupar em realizar a função social do estado, em detrimento de possíveis interesses financeiros. Como entrave gerado por esse senso, um dos pesquisadores apontou a falta de apoio institucional da própria universidade para fomentar projetos de pesquisa com possível aplicação de mercado.

Um dos pesquisadores entrevistados acrescenta que as empresas brasileiras não têm o aporte necessário para investir em inovação, por ser algo complexo e fora da realidade [econômica] farmacêutica local. Sendo assim, o problema estaria também na capacidade de absorção das novas tecnologias por parte das empresas.

Questões relativas à validação da tecnologia foram levantadas como barreira relacionada à orientação. A intensa regulação do setor farmacêutico exige procedimentos específicos para aprovação de novos produtos. Segundo os pesquisadores, a universidade é normalmente capaz de desenvolver pesquisas até o estágio pré-clínico. Contudo, é necessário ter equipamentos calibrados e as certificações laboratoriais exigidas para que o teste seja validado, de forma que a empresa não tenha que refazê-lo futuramente. Em congruência, o membro do sindicato de empresas afirmou que a transferência de tecnologias para empresas seria mais fácil se o pesquisador realizasse o desenvolvimento à luz das questões regulatórias e apresentasse sua oferta de produto já com registro e com segurança e eficácia comprovados. Essa mesma ótica foi abordada pelos integrantes da fundação pública de pesquisa e produção de medicamentos, que afirmaram que a pesquisa em escala de bancada não basta para analisar sua aplicação industrial. 


\subsection{Barreiras relacionadas à transação}

A burocracia como entrave ao estabelecimento de relação entre universidade-empresa foi citada de forma genérica por muitos entrevistados. Tanto pesquisadores quanto empresas concordam que a lentidão nos processos e registros para estabelecimento de parcerias é uma dificuldade para cooperação entre ambos os lados. No contexto da empresa de fitoterápicos, por exemplo, a parceria com a universidade seria benéfica para suas atividades de desenvolvimento de novos medicamentos pois, além da expertise dos pesquisadores da universidade e da utilização de sua infraestrutura laboratorial, essa relação é um meio de acesso ao fomento governamental. Contudo, a demora nos processos de contrato e liberação da verba foi citado como o principal desestímulo para possíveis aproximações.

Além disso, para os representantes da universidade, aspectos de conflito de interesses dentro da instituição também contribuem para a dificuldade em se estabelecer parcerias duradouras. Com a instabilidade política dos órgãos públicos, seus objetivos e focos de atuação se alteram, sendo que a cada nova administração diferentes projetos de pesquisa são priorizados.

Relativo à comunicação entre universidade e empresa, um dos pesquisadores relatou desconhecimento em como dar o primeiro passo para início da relação. Esse desconhecimento e inexperiência, principalmente de aspectos jurídicos, gera insegurança nos pesquisadores no estabelecimento das parcerias. Representantes da fundação pública afirmaram que muitos pesquisadores têm receio de transferir sua pesquisa para as empresas, pelo medo de não serem devidamente remunerados pelo seu trabalho. Um ponto abordado pela representante do NIT é a falta de alguém que entenda os riscos, linguagens e contingências desse tipo de parceria também na empresa.

A noção de confidencialidade sobre as publicações científicas emergiu mais timidamente nas entrevistas. Isso foi pontuado somente por um dos entrevistados do meio empresarial, que afirmou ser maléfica a publicação de trabalhos pelos professores sem antes consultarem os anseios da empresa quanto a propriedade intelectual e possibilidades de exploração comercial.

\subsection{Novas barreiras}

Em adição, foram mencionadas barreiras além daquelas previamente mapeadas na literatura. Primeiramente, foi destacado por todos entrevistados o alto grau de regulamentação e rigor da ANVISA, nos procedimentos do setor farmacêutico. Apesar dessa regulamentação não ser diretamente relacionada à formação de parceria entre universidade e empresa, torna-se uma barreira à medida que enrijece os processos do setor dificultando iniciativas de inovação ou melhoria por parte das empresas. Dois dos entrevistados relacionados à indústria citam a regulação excessiva em todos os âmbitos da empresa, como por exemplo complexidade documental necessária para a movimentação de maquinário dentro do ambiente produtivo, algo trivial em outros setores industriais. Por outro lado, vários entrevistados reafirmaram a importância da atual regulação para a segurança e qualidade dos medicamentos produzidos no país.

Ademais, o tema de compartilhamento de riscos foi recorrente nas entrevistas, contribuindo em muitas barreiras já mencionadas. Para investir em uma nova tecnologia, as empresas se baseiam em indicadores financeiros como custo de desenvolvimento e tempo de retorno de investimento. Sendo assim, almejam tecnologias em fase de desenvolvimento avançada, em que parte dos riscos já foram mitigados na pesquisa acadêmica e a previsibilidade das atividades que a separam de uma exploração comercial do produto é maior. Por outro lado, os pesquisadores consideram que há foco excessivo da indústria no curto prazo e baixo risco, e acredita-se que universidade não tem o papel e nem recursos para arcar com a lapidação da tecnologia sem o apoio financeiro da empresa. Sendo assim poucas empresas estariam 
dispostas a fomentar tecnologias em estágio inicial de desenvolvimento. Isso evidencia uma lacuna no processo de inovação quanto às tecnologias que as empresas conseguem absorver, e quais estão sendo oferecidas pela universidade. A partir das entrevistas, constatou-se que um forte gargalo desse processo está nos testes clínicos.

\section{CONCLUSÃO}

O trabalho buscou oferecer um diagnóstico inicial das barreiras da relação universidadeempresa, sob o ponto de vista de diferentes atores da indústria farmacêutica de Minas Gerais. A partir desse embasamento, torna-se possível a elaboração de pesquisas que auxiliem no desenvolvimento de políticas e ações de gestão específicas para o setor. Uma limitação importante está na ausência da perspectiva de agentes representantes do governo, dada a importância deste para o debate. Ressalta-se também as limitações referentes a amostra de pesquisa, principalmente pela quantidade de entrevistas realizadas.

Vale destacar possíveis ações sugeridas nas entrevistas como a proposição de um processo de gestão da parceria robusto (e específico para os desafios deste setor), que conte com interlocutores capacitados para este diálogo de ambos os lados. Quanto aos testes clínicos, uma alternativa sugerida seria a construção de centros de pesquisa especializados nas etapas de validação de novos produtos, a partir de parcerias entre empresas reduzindo os investimentos necessários.

Como continuidade deste estudo, prevê-se não somente o aumento do espectro de entrevistados, mas também ações diretas de apoio à transferência tecnológica sob o âmbito da pesquisa-ação que elucidem novos desafios e soluções de gestão aplicáveis a esta realidade.

\section{REFERÊNCIAS}

ALBUQUERQUE, E. da M.; CASSIOLATO, J. E. As especificidades do sistema de inovação do setor saúde. Revista de Economia Política, v. 22, n. 4, p. 88, 2002.

ANVISA, Agência Nacional de Vigilância Sanitária. Disponível em: <www.anvisa.gov.br>. Acesso em: 25 Mai. 2017

BASTOS, V. D. Inovação farmacêutica: padrão setorial e perspectivas para o caso brasileiro. BNDES Setorial, Rio de Janeiro, n. 22 , p. 271-296, set. 2005.

BITTENCOURT, P. F.; RAPINI, M. S.; PARANHOS, J. Reflexos locacionais na interação universidade-empresa nos setores químico e farmacêutico brasileiro. Ensaios FEE, v. 33, n. 2, 2012.

BRUNEEL, J.; D'ESTE, P.; SALTER, A. Investigating the factors that diminish the barriers to university-industry collaboration. Research policy, v. 39, n. 7, p. 858-868, 2010.

CASTILHO, J. et al. A methodology to build an initial R\&D portfolio for industry-university cooperation. In: Management of Engineering and Technology (PICMET), 2015 Portland International Conference on. IEEE, 2015. p. 257-269

DA MOTTA VIEIRA, V. M.; OHAYON, P. Inovação em fármacos e medicamentos: estadoda-arte no Brasil e políticas de P\&D. Revista Economia \& Gestão, v. 6, n. 13, 2008.

ETZKOWITZ, H. The triple helix: university-industry-government innovation in action. Routledge, 2008. 
GASSMANN, O.; REEPMEYER, G.; ZEDTWITZ, M. Leading Pharmaceutical Innovation: Trends and Drivers for Growth in the Pharmaceutical Industry. Springer-Verlag Berlin Heidelberg, 2008.

GRABOWSKI, H.; VERNON, J.; DIMASI, J. Returns on Research and Development for 1990s New Drugs Introductions. In: Pharmacoeconomics 20, Suppl. 3, pp. 11-29. 2002.

HASENCLEVER, L. A relação universidade-empresa e a inovação no Brasil: os casos do Porto Digital, da indústria farmacêutica e da UEG. Tese de Doutorado. Universidade Federal do Rio de Janeiro.

PALMEIRA FILHO, P. L.; PAN, S. S. K. Cadeia farmacêutica no Brasil: avaliação preliminar e perspectivas. BNDES Setorial, Rio de Janeiro, n. 18, p. 3-22, 2003.

PARANHOS, J. Interação entre empresas e instituições de ciência e tecnologia: o caso do sistema farmacêutico de inovação brasileiro. In: Interação entre empresas e instituições de ciência e tecnologia: o caso do sistema farmacêutico de inovação brasileiro. EdUERJ, 2012.

PARTHA, D.; DAVID, P. A. Toward a new economics of science. Research policy, v. 23, n. 5, p. 487-521, 1994

PAVITT, K. Sectoral patterns of technical change: towards a taxonomy and a theory. Research policy, v. 13, n. 6, p. 343-373, 1984.

PIMENTEL, V. P. et al. O desafio de adensar a cadeia de P\&D de medicamentos biotecnológicos no Brasil. BNDES Setorial, Rio de Janeiro, n. 38, 2013.

RADAELLI, V. A Nova Conformação Setorial da Indústria Farmacêutica Mundial: redesenho nas pesquisas e ingresso de novos atores. Revista Brasileira de Inovação, v. 7, n. 2 jul/dez, p. 445-482, 2008.

SCHUMPETER, J. The Theory of Economic Development. Cambridge: Harvard University Press, 1934.

SEGATTO-MENDES, A. P. Análise do processo de cooperação tecnológica universidadeempresa: um estudo exploratório. 1996. Tese de Doutorado. Universidade de São Paulo.

SINDUSFARMA - Sindicato da Indústria de Produtos Farmacêuticos no Estado de São Paulo. Relatório Anual de Atividades 2015. São Paulo, 2016.

WEBSTER, A.; ETZKOWITZ, H.. Academic-industry relations: The second academic revolution?: A framework paper for the proposed research workshop on academic-industry relations. London: Science Policy Support Group, 1991. 